\title{
Multiple Agent Roles in an Adaptive Virtual Classroom Environment
}

\author{
Gregor Mehlmann, Markus Häring, René Bühling, \\ Michael Wißner, and Elisabeth André \\ Augsburg University, Multimedia Concepts and their Applications, \\ D-86159 Augsburg, Germany \\ \{mehlmann, haering, buehling, wissner, andre\} @informatik. uni-augsburg.de \\ http://mm-werkstatt.informatik. uni-augsburg.de/
}

\begin{abstract}
We present the design of a cast of pedagogical agents impersonating different educational roles in an interactive virtual learning environment. Teams of those agents are used to create different learning scenarios in order to provide learners with an engaging and motivating learning experience. Authors can employ an easy to use multimodal dialog authoring tool to adapt lecture and dialog content as well as interaction management to meet their respective requirements.
\end{abstract}

\section{Motivation}

Embodied conversational agents are widely used in educational applications such as virtual learning and training environments [1]. Beside possible negative effects of virtual characters [2], there is empirical evidence that virtual pedagogical agents and learning companions can lead to an improved perception of the learning task and increase the learners' commitment to the virtual learning experience [3]. They can promote the learners' motivation and self-confidence, help to prevent or overcome negative affective states and minimize undesirable associations with the learning task, such as frustration, boredom or fear of failure. It has been shown that a one-sided coverage of knowledge transfer or the employment of only a single educational role may either lead to satisfying learning success or motivation, but usually not both at the same time [4]. The usage of multiple virtual characters personating different educational roles can have positive influence on both the learners' learning success and their engagement. Teams of pedagogical agents can help the learners to classify the conveyed knowledge and allow for a continuous reinforcement of beliefs [5].

Point of origin for our research is DynaLearn [6], an interactive learning environment in which learners can express their conceptual knowledge through qualitative reasoning models [7]. In this paper, we present our effort of enriching DynaLearn with a cast of virtual characters, aiming at increasing learners' motivation as well as their learning success. We considered a variety of teaching methods, learning strategies and ways of knowledge conveyance and verification. The integration of an easy to use authoring tool simplifies visual prototyping of didactic and narrative structure as well as the creation of lecture content. 


\section{Related Work}

The classical role of an agent in a learning environment is that of a teacher, see, for example, [8] and [1]. In [9], "AutoTutor" is presented, which allows learners to learn by having a very interactive natural language conversation with a talking head, the virtual tutor.

"Betty's Brain" [10] features Betty, a talking head, who is a so-called teachable agent. Learners can teach her by building a concept map and asking questions about it with the ultimate goal of preparing her for a quiz. Also, multiple teachable agents can compete in a quiz show like application. Questions are asked by a virtual quizmaster and each agent responds according to their concept map.

In [11], another virtual character in "Betty's Brain" is mentioned: Mr. Davis, the teacher (also realized as a talking head). Mr. Davis helps learners by giving guidelines about teaching in general or hints that address specific situations, but not the task or domain at hand. There is also no real interaction between Mr. Davis and Betty.

A closer look at these interactive learning environments shows that all of them feature a teacher-like character that interacts with the learner. However, how this interaction looks like and what is actually communicated differs widely. Only one of the systems makes use of a teachable character. Also, only one system features fully embodied agents, that can also communicate through gestures. Finally, only one of the systems features more than one character role. But these characters only interact with the learner and never with each other.

Taking all this together, we decided to integrate the following character roles into our learning environment: A Teacher who helps the learner with specific and general hints through an interactive dialog, a Teachable Agent who can be taught by the learner and a Quizmaster who adds a playful element by asking the learner questions, but who also directly interacts with the teachable agent to form a presentation team as suggested in [5].

\section{Character Role Design}

Animal characters act as culture-neutral personalities and are expected to have lower communication skills than human-like characters allowing users to more willingly forgive technical imperfections $[12,13]$. For these reasons, we decided to create a set of cartoonish hamster characters. We aim at ensuring that the agents' behavior follows approved pedagogical principles and meets the learners' expectations of the roles. For the definition of behavioral rules and patterns for our educational roles, we fall back on three established teaching methods from educational science: Learning by Teaching [10], Scaffolding [14,15] and Educational Quizzes [16].

\subsection{Learning by Teaching}

Learning by Teaching is an effect that many learners experience while learning with or teaching their peers. A Teachable Agent (TA) transfers this principle 

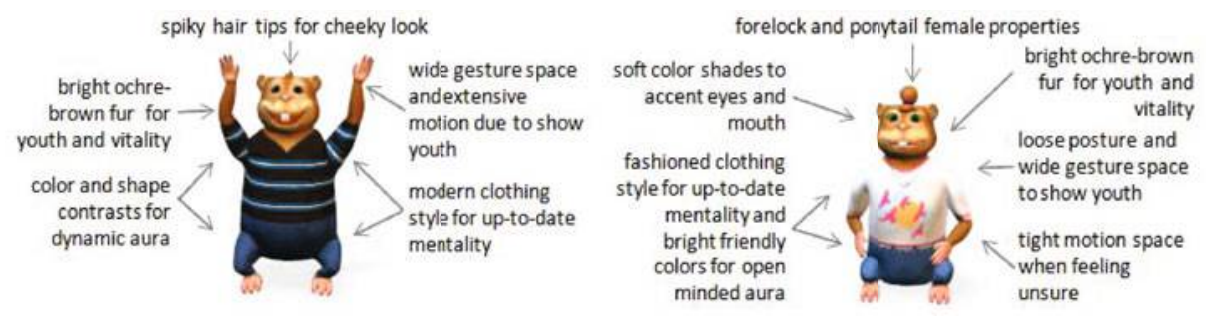

Fig. 1. The design criteria for the teachable boy and girl characters

to the field of learning environments. It has a knowledge representation that can be created by the learner. From this representation the agent can extract answers to questions asked by the learner. It is also able to explain its train of thought, so learners can see the causal chains in their own model. Testing the TA's understanding of the subject through questioning, learners can evaluate their own representation and find mistakes when the agent does not answer as expected.

Constant verification of one's own understanding is an important part in the learning process that unfortunately often comes short due to learners' aversion to tests. However, learners are less restrained in making an agent take a test multiple times. That is why we allow learners to take part in an educational quiz but to also send their TA instead. Since the TA's knowledge mirrors the learners' knowledge, it can serve as their proxy.

Though Learning by Teaching aims for learning success, the role of the teachable agent is more a motivating one. Following the research of Kim et al. [4] our TA forges a peer-like relationship to the learner with its low-competent behavior.

The learner is able to choose between a male and female personal pet and give it a name. Graphical details and animations differ and depend on the personality of each hamster's gender as shown in Figure 1. The boy character is designed to appear as a lively and extroverted person whereas politeness and open mindedness dominate the girl's personality.

\subsection{Instructional Scaffolding}

Of course we cannot expect the learners to detect and solve all problems, that occur during their work, by themselves. They need the help of more competent agents, like our teacher agent. We chose the constructivist approach of Scaffolding as learning principle for this role. Scaffolding emphasizes that learners should do as much work as possible by themselves. The teacher only provides assistance if learners do not possess the necessary skills or knowledge to solve the current problem.

The overview from Lipscomb et al. [14] and the work of Cade et al. [15] helped us identify the means for reaching this goal. While Cade et al. mention scaffolding as one of their mutual exclusive dialog modes in one-on-one tutoring sessions, 


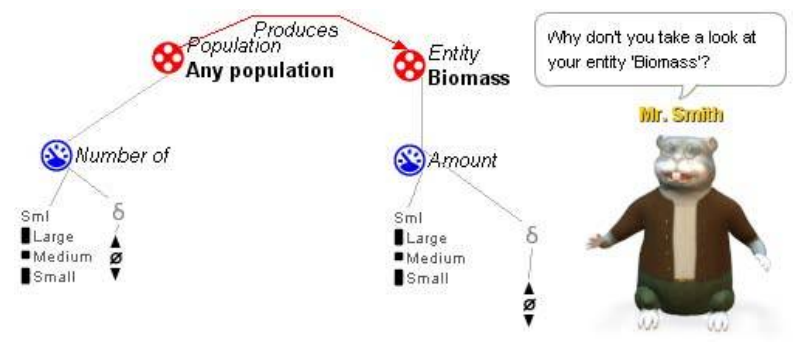

Fig. 2. The teacher giving a hint in the scaffolding phase

Lipscomb et al. describe scaffolding as a more extensive teaching principle. We combine the strength of both researches in our teacher role. The more widespread scaffolding of Lipscomb et al. determines the general behavior of our teacher, while we use dialog modes of Cade et al. as dialog steps within this behavior.

The task of our teacher is to communicate mistakes found in a learner's model and to help fixing them step-by-step. For this purpose the teacher provides different teaching aids for the learner from one of the following three categories: Lecture, Scaffolding and Modeling. The agent usually chooses with an equal chance between lecture and scaffolding when providing an aid. These chances are based on the observations of Cade et al. where lectures and scaffolding were the most present dialog modes with a very similar frequency. As we ideally want the learner to find the solution by himself, modeling (i.e. the exact correction of the mistake) is only appropriate if the teacher has exhausted all other means. In this way, we ensure learners can proceed with the correction of their model even if they cannot cope with a particular problem.

Figure 2 shows the teacher character giving a hint about wrong parts of a learner's model. His dialog texts convey a good-natured personality, as he never scolds the learner for mistakes or failures, but uses praise and reassurance. It can be seen that the teacher role is designed as an aged person having high competence and knowledge. To visually convey these aspects the teacher hamster and its traditional styled clothes are shaded with warm natural colors to suggest a calm aura and a down-to-earth mentality. Also round and soft facial shapes lead to a friendly charisma which is important for a trustful contact person to ask for help when needed. Age and experience are supported by bright gray colored fur, accessories like eyeglasses and calm animation characteristics.

\subsection{Educational Quizzes}

Tests are usually perceived as stressful situations having negative effects on concentration or motivation. In observations of quiz forms in several well known television quiz shows we found out that quizmasters sometimes try to loosen up such situations in order to countervail their negative effects and to provide an enjoyable form of test. They start lively conversations with their candidates and 


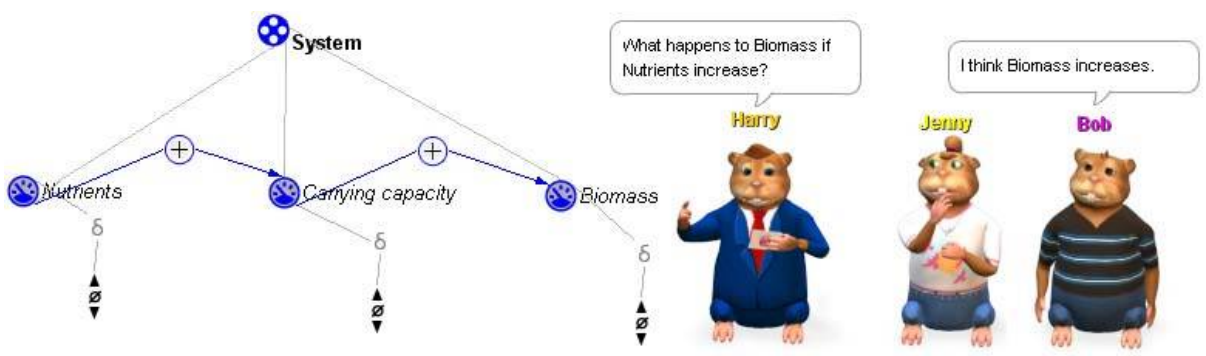

Fig. 3. Quizmaster and two teachable agents in an educational quiz scenario

discuss topics that are familiar to the participants such as job or leisure activities. We mimic this behavior with our quizmaster character. Beside the quizmaster's general behavior of asking questions and giving feedback, we integrate smalltalk utterances into the dialog. They serve as short, preferable humorous distraction for the participant that actually have no connection to the current quiz domain. As mentioned above, the quizmaster can engage a human learner as well as their teachable agent. Also, a quiz with more than one participating teachable agent is possible.

Figure 3 shows the quizmaster and the two teachable agents. To adapt the idea of a calming "fun manager" the quizmaster is designed to appear as a strongly organized, up to date person who is entertaining and serious at the same time. Seriousness and maturity are reflected in darker shaded fur and highly saturated clothing colors.

\section{Modeling Dialog and Interaction}

The dialog and interaction management for our agents is realized with the authoring tool SceneMaker [17], which facilitates the creation of interactive performances because it divides the authoring task into the creation of dialog content and the modeling of the narrative and didactic structure of a lecture.

Dialog content is organized in a set of parameterizable scenes that are specified in a multimodal scenescript which resembles a movie script with dialog utterances and stage directions for controlling gestures, postures and facial expressions. For each scene, we provide a number of variations, subsumed in a scenegroup, to increase variety and to avoid repetitive behavior that would impact the agents' believability. As shown in Figure 4, scenescript content is created both manually (a) and by an external generation module (b) that generates question phrases from a qualitative reasoning model.

The narrative structure of a lecture is controlled by a sceneflow - a hierarchical and concurrent finite-state machine specifying the logic organization and temporal order in which scenes are played. A state in a sceneflow may enfold several subgraphs, thus allowing for the hierarchical refinement and parallel decomposition of the sceneflow. This feature allows us to carry out the modeling 


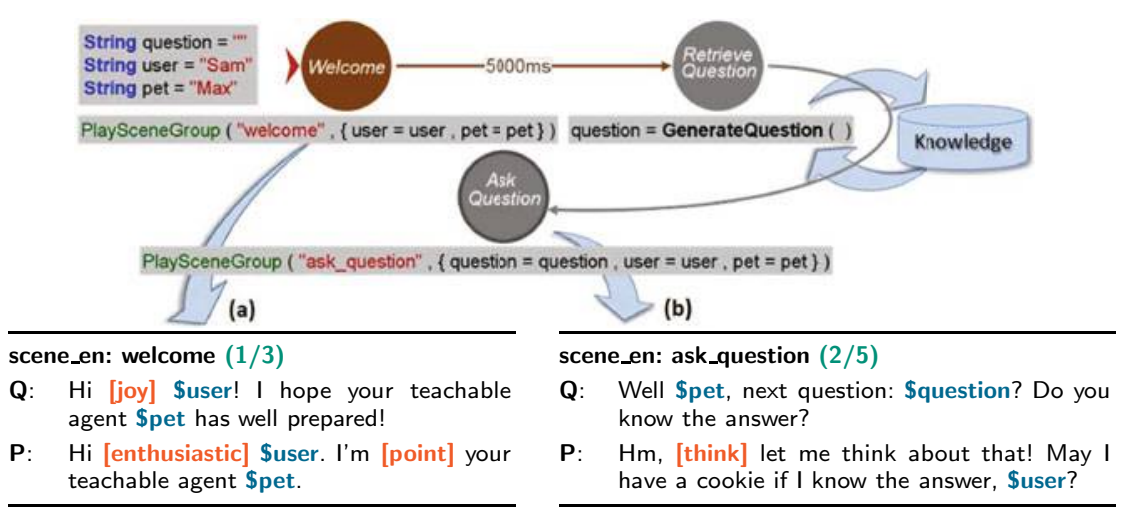

Fig. 4. Examples of calling scenes of the multimodal scenescript

task in a divide-and-conquer manner: Except for synchronization measures, various control processes and user input processing as well as the individual agents' behavior are modeled independently of each other. Thus, we keep the sceneflow clearly arranged, extensible and parts of it exchangeable.

The sceneflow interpreter allows the real-time visualization of a sceneflow's execution and active scenes within the graphical user interface in order to test, simulate and debug a model, to control the modeling progress and to verify the correctness of the model.

\section{Discussion}

An evaluation of the virtual characters' appearances confirmed that they successfully conveyed their intended roles and meaning. Another pilot evaluation of subjective measures confirmed that learners enjoyed the interaction with our pedagogical agents and perceived the virtual classroom setting as engaging and motivating. They understood the different learning scenarios and the justification for each single educational role. They felt that all agents and their respective educational roles successfully helped learning. More detailed and exhaustive evaluations will have to show whether those results also apply to objective measures such as learning success and if the educational roles created in the context of DynaLearn can also be applied to other learning environments.

\section{Acknowledgments}

This work is supported by DynaLearn, co-funded by the EC within the 7th Framework Programme, Project no. 231526 (http://www.DynaLearn.eu).

\section{References}

1. Johnson, L.W., Rickel, J.W.: Animated Pedagogical Agents: Face-to-Face Interaction in Interactive Learning Environments. Artificial Intelligence in Education (11), 47-78 (2000) 
2. Rickenberg, R., Reeves, B.: The Effects of Animated Characters on Anxiety, Task Performance and Evaluations of User Interfaces. In: Proc. of the SIGCHI Conf. on Human Factors in Computing Systems, pp. 49-56. ACM, New York (2000)

3. van Mulken, S., André, E., Müller, J.: The Persona Effect: How Substantial Is It? In: Proc. of HCI on People and Computers XIII, pp. 53-66. Springer, London (1998)

4. Kim, Y., Baylor, A.L.: PALS Group: Pedagogical Agents as Learning Companions: The Role of Agent Competency and Type of Interaction. Educational Technology Research and Development 54(3), 223-243 (2006)

5. André, E., Rist, T., van Mulken, S., Klesen, M., Baldes, S.: The Automated Design of Believable Dialogues for Animated Presentation Teams. In: Embodied Conversational Agents, pp. 220-255. MIT Press, Cambridge (2001)

6. Bredeweg, B., Gómez-Pérez, A., André, E., Salles, P.: DynaLearn - Engaging and Informed Tools for Learning Conceptual System Knowledge. In: Technical Report FS-09-02, pp. 46-51. AAAI Press (2009)

7. Bredeweg, B., Linnebank, F., Bouwer, A., Liem, J.: Garp3 - Workbench for Qualitative Modelling and Simulation. Ecological Informatics (4), 263-281 (2009)

8. Conati, C., Zhao, X.: Building and Evaluating an Intelligent Pedagogical Agent to Improve the Effectiveness of an Educational Game. In: Proc. of the 9th Int. Conf. on Intelligent User Interfaces, pp. 6-13. ACM, New York (2004)

9. Graesser, A.C., Person, N.K., Harter, D.: The Tutoring Research Group: Teaching Tactics and Dialog in Autotutor. Artificial Intelligence in Education (12), 257-279 (2001)

10. Blair, K., Schwartz, D., Biswas, G., Leelawong, K.: Pedagogical Agents for Learning by Teaching: Teachable Agents. In: Educational Technology \& Society, Special Issue on Pedagogical Agents (2006)

11. Biswas, G., Roscoe, R., Jeong, H., Sulcer, B.: Promoting Self-Regulated Learning Skills in Agent-Based Learning Environments. In: Proc. of the 17th Int. Conf. on Computers in Education, pp. 67-74 (2009)

12. Mateas, M.: An Oz-Centric Review of Interactive Drama and Believable Agents. In: Technical Report CMU-CS-97-156, School of Computer Science CMU (1997)

13. Bredeweg, B., Liem, J., Linnebank, F., Bühling, R., Wißner, M., Gracia del Río, J., Salles, P., Beek, W., Gómez Pérez, A.: DynaLearn: Architecture and Approach for Investigating the Acquisition of Conceptual System Knowledge for Ill-Defined Domains. In: Aleven, V., Kay, J., Mostow, J. (eds.) Intelligent Tutoring Systems. LNCS, vol. 6095, pp. 272-274. Springer, Heidelberg (2010)

14. Lipscomb, L., Swanson, J., West, A.: Scaffolding. In: Emerging Perspectives on Learning, Teaching and Technology (2001)

15. Cade, W.L., Copeland, J.L., Person, N.K., D'Mello, S.K.: Dialogue Modes in Expert Tutoring. In: Woolf, B.P., Aïmeur, E., Nkambou, R., Lajoie, S. (eds.) ITS 2008. LNCS, vol. 5091, pp. 470-479. Springer, Heidelberg (2008)

16. Randel, J.M., Morris, B.A., Wetzel, C.D., Whitehill, B.V.: The Effectiveness of Games for Educational Purposes: A Review of Recent Research. Simulation and Gaming (23), 261-276 (1992)

17. Gebhard, P., Kipp, M., Klesen, M., Rist, T.: Authoring Scenes for Adaptive, Interactive Performances. In: Proc. of the 2th Int. Conf. on Autonomous Agents and Multiagent Systems, pp. 725-732. ACM, New York (2003) 